01

\title{
Функция Нордгейма в массиве однородных идентичных нанотрубок при полевой эмиссии
}

\author{
(C) Н.Р. Садыков, С.Е. Жолниров, И.А. Пилипенко
}

Снежинский физико-технический институт Национального исследовательского ядерного института МИФИ, 456776 Снежинск, Челябинской обл., Россия

e-mail: n.r.sadykov@rambler.ru

Поступило в Редакцию 26 декабря 2020 г.

В окончательной редакции 11 февраля 2021 г.

Принято к публикации 2 марта 2021 г.

Рассмотрена задача полевой эмиссии на основе углеродных нанотрубок. Получена функция прохождения частиц. Установлена зависимость функции Нордгейма от длины наночастиц. Рассмотрен предельный переход для величины коэффициента прохождения для полевой эмиссии с поверхности катода при отсутствии на его поверхности наночастицы.

Ключевые слова: полевая эмиссия, функция Нордгейма, углеродные нанотрубки.

DOI: $10.21883 /$ JTF.2021.07.50948.350-20

\section{Введение}

Самой простой формой для исследования электронного транспорта являются удлиненные карбины как одномерные (кумуленовые и полииновые цепочки [1-4], так и двумерные структуры (нанотрубки, наноленты [5-7]). К этим структурам применимы такие понятия, как кинетическая индуктивность $\tilde{L}_{k}$, квантовая емкость $C_{Q}$ и активное сопротивление на единицу длины $R_{a}[8,9]$ :

$$
\begin{gathered}
\tilde{L}_{k}=h /\left(8 e^{2} v_{F}\right), \quad C_{Q}=8 e^{2} /\left(h v_{F}\right), \\
R_{a}=h /\left(2 e^{2}\right) \approx 12.9 \mathrm{k} \Omega,
\end{gathered}
$$

которые имеют заведомо квантовую природу. Например, если длина углеродной нанотрубки меньше одного микрометра $L \leq 1 \mu \mathrm{m}$, то активное сопротивление равно $R_{a}$. Если больше одного микрометра, то сопротивление НТ пропорционально длине $R_{\mathrm{CNT}}=L R_{a}$, т. е. существует прямая аналогия с классической физикой. Таким образом, электронный транспорт в наноматериалах осуществляется по баллистическому механизму: электрон, не теряя энергии, туннелируется сквозь нанообъект без рассеяния на колебаниях решетки, примесях или дефектах на расстояния до $\approx 1 \mu \mathrm{m}$. Баллистический транспорт заряда в наноматериалах обусловлен тем, что обычные размеры нанопровода гораздо меньше, чем длина свободного пробега электронов. Длину свободного пробега электронов можно оценить следующим образом: скорость электронов на поверхности Ферми $v_{\mathrm{F}} \sim 10^{6} \mathrm{~m} / \mathrm{s}$, время релаксации $\tau \sim 10^{-12} \mathrm{~s}$, длина свободного пробега $\lambda \approx v_{\mathrm{F}} \tau \sim 1 \mu \mathrm{m}$

Уникальность удлиненных карбинов состоит в том, что их поперечные размеры на три-четыре порядка могут быть меньше продольных размеров, что приводит к очень малым значениям коэффициента деполяризации вдоль наночастиц. Данная закономерность актуальна в эффекте полевой эмиссии, где необходимы большие значения напряженности электрического поля [10]. В настоящей работе рассмотрим совокупность вытянутых параллельных зигзагообразных нанотрубок (УНТ), ориентированных вдоль поля. Чтобы исключить взаимное влияние наночастиц, рассмотрим случай, когда взаимодействие между нанотрубками не учитывается. Для этого расстояние между соседними УНТ должно быть равно или больше длины нанотрубки. Такие материалы существуют и в настоящее время, например, в [11], чтобы сделать катод на подложке перпендикулярно поверхности, УНТ выращивают так, чтобы расстояние между соседними УНТ равнялось удвоенной длине УНТ. Это соответствует минимальному влиянию окружающей среды на излучение отдельных УНТ и дает максимальное излучение катодного тока. Автоэмиссионные свойства ориентированных пучков однослойных УНТ, декорированных наноалмазами, экспериментально исследованы в работе [12].

В настоящей работе покажем, что использование массива параллельных друг другу идентичных нанотрубок в эффекте полевой эмиссии приводит к тому, что функция Нордгейма начинает зависеть от геометрии нанотрубки.

На рис. 1 схематично изображен массив углеродных нанотрубок (УНТ) длиной в несколько нанометров.

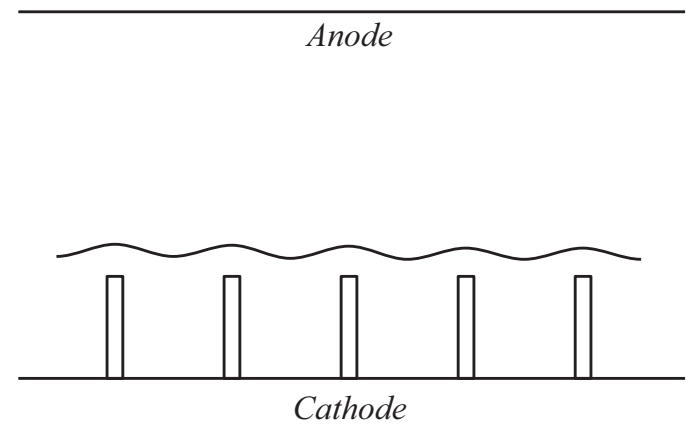

Pис. 1. Схема массива УНТ длиной в несколько нанометров. 


\section{1. Электродинамика УНТ. Формула Ландауера для нанотрубок}

Транспорт электронов в наноматериалах определяется баллистическим механизмом, когда длина наночастицы намного меньше длины свободного пробега электронов. В этом случае для расчета тока можно использовать формулу Ландауэра $[13,14]$

$$
I_{1}(V, T)=2 e \int\left[f_{L}(E, T)-f_{R}(E, T)\right] \tau(E, V) \frac{v_{z} d p_{x}}{2 \pi \hbar},
$$

где $V$ - разность потенциалов между правым $(R)$ или левым $(L)$ электродами (между катодом и анодом), $e-$ заряд электрона, постоянная Планка, а $\tau-$ функция пропускания, зависящая от энергии $E$ носителей заряда, температуры $T$ и разности потенциалов $V$. Функции $f_{L}(E, T)$ и $f_{R}(E, T)$ определяют распределение Ферми

$$
f_{L, R}(E, T)=\frac{1}{\exp \left(\frac{E-U_{1}-\mu_{L, R}}{k T}\right)+1},
$$

где $\mu_{L}=0, \mu_{R}=-|e V|-$ изменение химического потенциала катода и анода соответственно. В (2) величина $\left(v_{z} / h\right) d p_{z}$, где $h=2 \pi \hbar$, определяет число состояний, которые пересекают поперечное сечение проводника за единицу времени в одномерном случае. Фактор 2 в (2) является в соответствии с принципом Паули следствием двух степеней свободы электрона на каждом уровне энергии. В (2) при интегрировании можно перейти от продольной компоненты импульса $p_{z}$ к новой переменной $E$ в соответствии с формулой

$$
v_{z} d p_{z}=d E \text {. }
$$

\section{2. Коэффициент прохождения при полевой эмиссии с острия удлиненных наночастиц}

Для определения тока в случае полевой эмиссии с острия наночастиц в соответствии с формулой (2) необходимо знать величину коэффициента прохождения (функции прохождения) частицы от анода к катоду. Для определения коэффициента прохождения носителей зарядов через потенциальную яму нужно знать профиль потенциала, который, в свою очередь, зависит от параметров внешнего электрического поля. В нашем случае нанотрубка находится на поверхности металлического катода с уровнем Ферми $E_{\mathrm{F}}=U_{1}$ (на рис. $2, a$ левая область $z<0, z-$ продольная координата). При отсутствии электрического поля $W=0$ потенциальная энергия будет иметь вид сплошной кривой, изображенной на рис. $2, a$. При наличии электрического поля $W \neq 0$ в области $z \geq 0$ потенциальная энергия будет содержать также потенциальную энергию внешнего потенциального поля $U_{\exp }=-|e| W z$ (см. рис. 2,b), где пренебрегаем наличием сил зеркального изображения. В этом случае внутри металлического катода $z<0$ электрическое поле отсутствует, поэтому там потенциал равен постоянной величине. В дальнейшем для упрощения расчетов полевой эмиссии $(W \neq 0)$ потенциал, изображенный на рис. $2, b$, аппроксимируем потенциалом, изображенным на рис. $2, c$ сплошной линией, где $U_{3 w}=-|e| W L_{1}$. Такая аппроксимация потенциальной энергии означает, что волновую функцию в нанотрубке мы должны аппроксимировать волновой функцией в мелкой потенциальной яме прямоугольной формы и использовать закон дисперсии для свободных электронов. Параметры потенциальной энергии, изображенной на рис. 2, $c$, приведены также в разд. 4.

Зная функцию потенциала для носителей заряда, получим в квазиклассическом приближении аналитическое решение для коэффициента прохождения. Для этого найдем решение стационарного уравнения Шредингера в области потенциала $z>L_{1}$, изображенного на рис. $2, a$ :

$$
-\frac{\hbar^{2}}{2 m} \frac{\partial^{2} \psi}{\partial z^{2}}+U \psi=E \psi
$$

где

$$
U(z)= \begin{cases}U_{1}, & z<0 \\ U_{2 a}, & 0 \leq z \leq L_{1}, \\ -|e| W z, & z>L_{1}\end{cases}
$$

Решение уравнения (5) в треугольной потенциальной яме в трех областях запишем в виде плоских волн и функции $\chi(z)$

$$
\psi(z)= \begin{cases}A_{1} e^{i k_{1} z}+B_{1} e^{-i k_{1} z}, & z<0, \\ A_{2} e^{i k_{2} z}+B_{2} e^{-i k_{2} z}, & 0 \leq z \leq L_{1}, \\ C \chi(z), & z \geq L_{1},\end{cases}
$$

где функция $\chi(z)$ выражается через функции Эйри $([15,16])$. Для волновой функции $\psi(z)$ из (7), исходя из условия неразрывности волновой функции и неразрывности плотности потока $S_{z}=$ $=i \hbar /(2 m)\left(\psi \partial \psi^{*} / \partial z-\psi^{*} \partial \psi / \partial z\right) \quad$ носителей $\quad$ заряда на границах разрыва потенциальной энергии из (6), выпишем граничные условия в явном виде

$$
\begin{gathered}
\left.\psi\right|_{z=-0}=\left.\psi\right|_{z=+0},\left.\quad \psi\right|_{z=L_{1}-0}=\left.\psi\right|_{z=L_{1}+0}, \\
\left.\frac{\partial \psi}{\partial z}\right|_{z=-0}=\left.\frac{\partial \psi}{\partial z}\right|_{z=+0},\left.\quad \frac{\partial \psi}{\partial z}\right|_{z=L_{1}-0}=\left.\frac{\partial \psi}{\partial z}\right|_{z=L_{1}+0},
\end{gathered}
$$

где в выражении для плотности потока $\psi^{*}$ является комплексно сопряженной величиной волновой функции, $m$ - масса носителей заряда.

Функция Эйри является решением уравнения

$$
\frac{\partial^{2} \psi}{\partial \xi^{2}}-\xi \psi=0
$$

где

$$
\xi=\left(\frac{2 m|e| W}{\hbar^{2}}\right)^{1 / 3}\left(L_{2}-z\right), \quad L_{2}=-E /(|e| W) .
$$



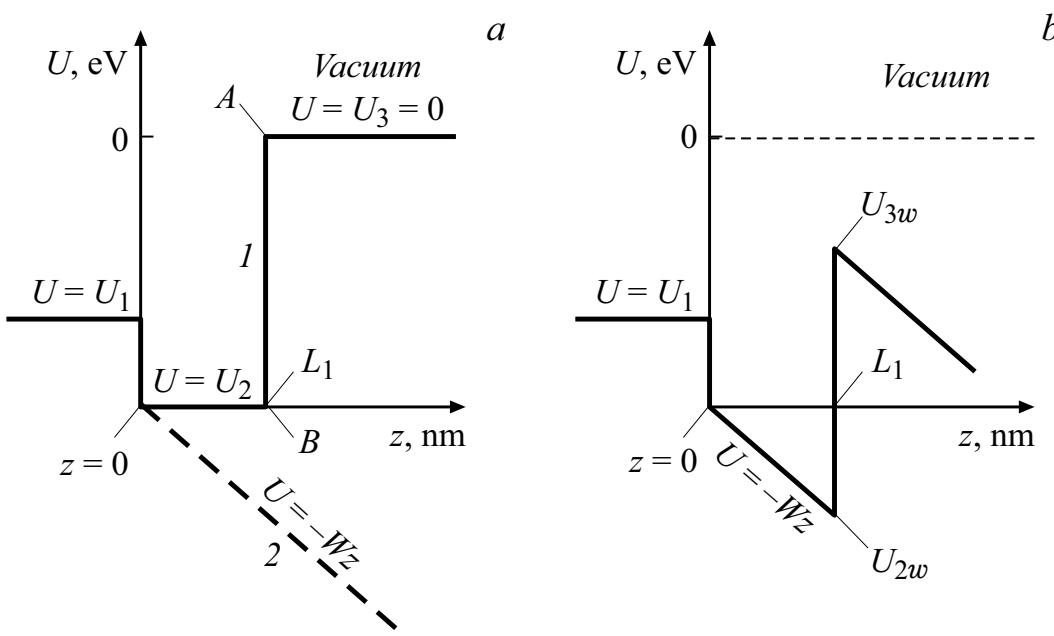

$b$

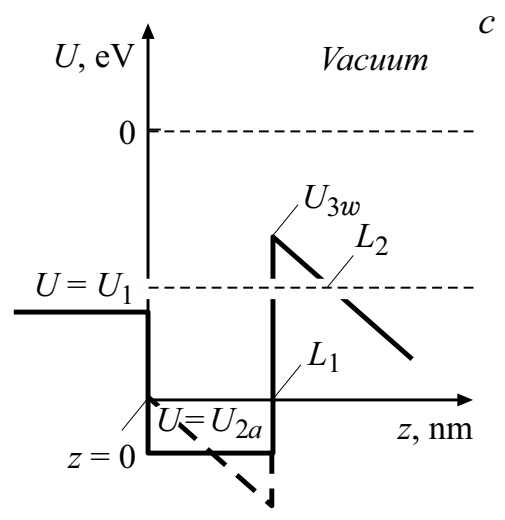

Рис. 2. Вид потенциальной ямы и аппроксимация распределения потенциала под действием однородного электрического поля

Решение уравнения (9) выражается через интеграл

$$
\chi(z)=\frac{1}{2 \pi} \int_{\gamma} \exp \left[-i\left(\frac{u^{3}}{3}+\xi u\right)\right] d u,
$$

где контур $\gamma$ определяет вид конкретного решения уравнения (9).

В дальнейшем нам понадобится решение уравнения Шредингера в области $z \geq L_{2}$ в однородном электрическом поле (6), имеющем асимптотику вида $\chi \sim \exp \left(i 2|\xi|^{3 / 2} / 3\right)$. Такое приближенное в окрестности точки $z=L_{2}$ решение можно получить, проведя интегрирование (11) по мнимой оси от $+i \infty$ до 0 и по вещественной оси от 0 до $+\infty([16])$

$$
\chi(z)= \begin{cases}\frac{-i}{2 \sqrt{\pi}|\xi|^{1 / 4}} \exp \left[i\left(\frac{2}{3}|\xi|^{3 / 2}+\frac{\pi}{4}\right)\right], & \xi<0, \\ \frac{-i}{2 \sqrt{\pi} \xi^{1 / 4}} \exp \left(\frac{2}{3} \xi^{3 / 2}\right), & \xi>0 .\end{cases}
$$

В работах $[17,18]$, исходя из двухточечной элементарной ячейки для металлических зигзаг-нанотрубок, и в работе [19], исходя из четырехточечной модели для узких металлических нанолент с крессельными краями, были получены собственные функции и собственных значения:

$$
\begin{gathered}
\psi \sim \exp ( \pm i \tilde{\chi}(z)), \quad \tilde{\chi}(z)=\left(\varepsilon+e W_{0} z\right)^{2} /\left(2 \hbar v_{\mathrm{F}} e W_{0}\right), \\
\varepsilon_{n}=E_{n}-E_{\mathrm{F}}=\frac{\pi \hbar v_{\mathrm{F}}}{2 L_{1}}(1+2 n),
\end{gathered}
$$

где $n=0, \pm 1, \pm 2, \pm 3, \ldots, E_{n}$ - эквидистантные уровни энергии заряженных частиц в нанотрубке, $E_{\mathrm{F}}-$ уровень Ферми нанотрубки (в нашей модели $E_{\mathrm{F}}=U_{2 a}$; (рис. $\left.2, c\right)$, $v_{\mathrm{F}}$ - скорость Ферми, напряженность продольного электрического поля, $\tilde{\chi}(z)$ - фаза. В работах $[17,18]$ при выводе формулы (13) на концах нанотрубок были сформулированы первые краевые условия. Поскольку в разд. 2 (перед рис. 2) мы сказали, что аппроксимируем потенциальную энергию, изображенную на рис. $3, b$, прямоугольной потенциальной ямой (сплошная кривая на рис. 2,c), волновую функцию в нанотрубке из (13) мы должны аппроксимировать волновой функцией в мелкой потенциальной яме прямоугольной формы и использовать закон дисперсии для свободных электронов. Поэтому в настоящей работе положим, что в отличие от [3] закон дисперсии для носителей заряда в области $z<0$ и $0 \leq z<L_{1}$ имеет вид

$$
k_{1}=\sqrt{2 m\left|E-U_{1}\right|} / \hbar, \quad k_{2}=\sqrt{2 m\left|E-U_{2 a}\right|} / \hbar
$$

соответственно. Из (7) с учетом граничных условий (9) на границах раздела $z=0$ и $z=L_{1}$ потенциала получим систему из четырех уравнений

$$
\begin{gathered}
A_{1}+B_{1}=A_{2}+B_{2}, \\
A_{1} k_{1}-B_{1} k_{1}=A_{2} k_{2}-B_{2} k_{2}, \\
A_{2} e^{i k_{2} L_{1}}+B_{2} e^{-i k_{2} L_{1}}=C \chi\left(L_{1}\right), \\
i A_{2} k_{2} e^{i k_{2} L_{1}}-i B_{2} k_{2} e^{-i k_{2} L_{1}}=\left.C\left(\frac{\partial \chi}{\partial z}\right)\right|_{z=L_{1}} .
\end{gathered}
$$

Система уравнений (15) позволяет получить коэффициент прохождения в зависимости от энергии частицы. Пусть слева из области $z<0$ падает плоская волна с амплитудой $A_{1}=1$, а в правой части вдали от особой точки $z=L_{2}$ (область $z>L_{2}$, т.е. $\xi<0$ в (12)) распространяется только бегущая вправо плоская волна. В результате вдали от особой точки $z>L_{2}$ для коэффициента прохождения из (15) получаем выражение

$$
\tau=\left.\left|\frac{C}{A_{1}}\right|^{2} \frac{1}{k_{1}}\left(|\chi|^{2} \frac{d \eta}{d z}\right)\right|_{z>L_{2}},
$$

где $\eta=2|\xi|^{3 / 2} / 3$; при выводе коэффициента прохождения $\tau$ учтено, что плотность потока $S_{z}$ в области $z>L_{2}$ 
определяется в соответствии с равенством ([20])

$$
\begin{array}{r}
S_{z}=\left.i \frac{\hbar}{2 m}|C|^{2}\left(\chi \frac{\partial \chi^{*}}{\partial z}-\chi^{*} \frac{\partial \chi}{\partial z}\right)\right|_{z>L_{2}} \\
=\left.\frac{\hbar}{m}|C|^{2}|\chi|^{2}\left[\frac{d(\eta)}{d z}\right]\right|_{z>L_{2}},
\end{array}
$$

а плотность потока падающей волны определяется равенством $S_{z}=\hbar k_{1}\left|A_{1}\right|^{2} / m$. Величина $\left|C / A_{1}\right|^{2}$ в равенстве (16) может быть вычислена в соответствии с формулой Крамера

$$
C=\frac{\left|\begin{array}{cccc}
1 & -1 & -1 & -A_{1} \\
-k_{1} & -k_{2} & k_{2} & -k_{1} A_{1} \\
0 & e^{i k_{2} L_{1}} & e^{-i k_{2} L_{1}} & 0 \\
0 & i k_{2} e^{i k_{2} L_{1}} & -i k_{2} e^{-i k_{2} L_{1}} & 0
\end{array}\right|}{\left|\begin{array}{cccc}
1 & -1 & -1 & 0 \\
-k_{1} & -k_{2} & k_{2} & 0 \\
0 & e^{i k_{2} L_{1}} & e^{-i k_{2} L_{1}} & -\chi\left(L_{1}\right) \\
0 & i k_{2} e^{i k_{2} L_{1}} & -i k_{2} e^{-i k_{2} L_{1}} & -\left.\left(\frac{\partial \chi}{\partial z}\right)\right|_{z=L_{1}}
\end{array}\right|} .
$$

Из (18) следует

$$
\begin{aligned}
\frac{C}{A_{1}}=\frac{2 i k_{1} k_{2}}{k_{2}\left[\left.\frac{d \chi}{d z}\right|_{z=L_{1}} \cos \left(k_{2} L_{1}+k_{2} \chi\left(L_{1}\right) \sin \left(k_{2} L_{1}\right)\right]+\right.} \\
+i k_{1}\left[-\left.\frac{d \chi}{d z}\right|_{z=L_{1}} \sin \left(k_{2} L_{1}\right)+k_{2} \chi\left(L_{1}\right) \cos \left(k_{2} L_{1}\right)\right]
\end{aligned}
$$

При выполнении условий $\xi \gg 1$ и $z \rightarrow L_{1}$ из (12) с учетом (10) следует равенство

$$
\begin{aligned}
& \left.\frac{d \chi}{d z}\right|_{z=L_{1}} \approx-\left[\frac{\sqrt{2 m|e| W\left(L_{2}-L_{1}\right)}}{\hbar}\right] \chi\left(L_{1}\right)=-\kappa \chi\left(L_{1}\right), \\
& \chi\left(L_{1}\right)=\frac{-i}{\left.2 \sqrt{\pi} \xi^{1 / 4}\right|_{z=L_{1}}} \exp \left[\frac{2 \sqrt{2 m|e| W}}{3 \hbar}\left(L_{2}-L_{1}\right)^{3 / 2}\right],
\end{aligned}
$$

где введено новое обозначение

$$
\kappa=\sqrt{2 m|e| W\left(L_{2}-L_{1}\right)} / \hbar .
$$

С учетом (20) преобразуем равенство (19) к виду

$$
\begin{aligned}
&\left.\left|\frac{C}{A_{1}}\right|^{2}\right|_{z=L_{1}}= \frac{16 \pi k_{1}^{2} k_{2}^{2}\left[\left(2 m|e| W / \hbar^{2}\right)^{1 / 6}\left(L_{2}-L_{1}\right)^{1 / 2}\right]}{k_{2}^{2}\left(-\kappa \cos \left(k_{2} L_{1}\right)+k_{2} \sin \left(k_{2} L_{1}\right)\right)^{2}+} \\
&+k_{1}^{2}\left(\kappa \sin \left(k_{2} L_{1}\right)+k_{2} \cos \left(k_{2} L_{1}\right)\right)^{2} \\
& \times \exp \left[-\frac{4 \sqrt{2 m|e| W}}{3 \hbar}\left(L_{2}-L_{1}\right)^{3 / 2}\right]
\end{aligned}
$$

Окончательно из (16) с учетом (22) получаем выражение для коэффициента прохождения

$$
\begin{aligned}
\tau=\frac{2 \kappa k_{2}}{\kappa^{2}+k_{2}^{2}} & \frac{2 k_{1} k_{2}}{k_{1}^{2}+\left(k_{2}^{2}-k_{1}^{2}\right) \sin ^{2}\left(k_{2} L_{1}-\varphi_{0}\right)} \\
& \times \exp \left[-\frac{4 \sqrt{2 m|e| W}}{3 \hbar}\left(L_{2}-L_{1}\right)^{3 / 2}\right],
\end{aligned}
$$

где при выводе учтено равенство

$$
\left.\left.\frac{1}{k_{1}}\left(|\chi|^{2} \frac{d \eta}{d z}\right)\right|_{z>L_{2}}=\frac{1}{4 \pi k_{1}} \frac{2 m|e| W}{\hbar^{2}}\right)^{1 / 3}, \varphi_{0}=\arctan \left(\kappa / k_{2}\right) .
$$

\section{3. Предельный переход при полевой эмиссии для коэффициента прохождения при наличии наночастиц нанометровой длины}

При выполнении условия $L_{1} \ll L_{2}, k_{2} \rightarrow k_{1}$ из равенства (23) получаем предельный переход для коэффициента прохождения для заряженной частицы с треугольным профилем потенциальной энергии (область туннелирования $0 \leq z \leq L_{2}$ )

$$
\tau=\frac{4 \sqrt{|E|} \sqrt{E-U_{1}}}{\left|U_{1}\right|} \exp \left[-\frac{4 \sqrt{2 m|E|^{4 / 2}}}{3 \hbar|e| W}\right],
$$

где при выводе (23) не учитывался поперечный разброс электронов при вылете с острия нанотрубок (с поверхности металла). Коэффициент прохождения из (25) совпадает с результатами задач, приведенными в [21] (задача 2.53 или задача 9.32). Таким образом, в нашей рассмотренной задаче существует предельный переход к случаю, когда полевая эмиссия происходит с поверхности металла при отсутствии наночастиц.

Ситуация меняется для величины коэффициента прохождения при наличии нанотрубки хоть и малой, но конечной длины $L_{1}$. В этом случае величина коэффициента прохождения в соответствии с (23) зависит от величины набега фазы $\Delta \varphi=k_{1} L_{1}$ вдоль зигзаг-нанотрубки с металлическим типом проводимости. Наибольшее значение коэффициента прохождения достигается при выполнении условия $\sin ^{2}\left(k_{2} L_{1}-\varphi_{0}\right)=0$, т. е. при величине набега фазы

$$
\Delta \varphi=k_{2} L_{1}=\varphi_{0}+\pi n, \quad n=1,2,3, \ldots,
$$

где величина $\varphi_{0}$ определена в (24).

В равенствах (23) и (26) волновое число $k_{2}$ и набег фазы были получены исходя из закона дисперсии для свободных электронов. В работах [17-19] |bf(cм. формулу (13)) функциональная зависимость набега фазы $\Delta \tilde{\gamma}$ и уровней энергии $\varepsilon_{n}$ для стационарных состояний заряженных частиц в зигзаг-нанотрубке и крессельных нанолентах при наличии продольного постоянного электрического поля были получены на основе модели двухточечной и четырехточечных элементарных ячеек

$$
\begin{aligned}
\Delta \tilde{\chi} & =\tilde{\chi}_{n, 2}-\tilde{\chi}_{n, 1}=\frac{\pi}{2}(1+2 n) \Leftrightarrow \varepsilon_{n}=E_{n}-E_{\mathrm{F}} \\
& =\frac{\pi \hbar v_{\mathrm{F}}}{2 L}(1+2 n),
\end{aligned}
$$

где $\tilde{\chi}_{n, 2}=\tilde{\chi}(z=L), \tilde{\chi}_{n, 1}=\tilde{\chi}(z=0), L-$ длина нанотрубки, фаза $\tilde{\chi}(z)$ определена в выражении (13). При 
выводе (27) на концах нанотрубок в работах $[19,20]$ были сформулированы первые краевые условия. Из (27) следует, что набег фазы носителей заряда и эквидистантные уровни энергии при наличии продольного постоянного электрического поля имеют одинаковую функциональную зависимость от квантового числа $n$. При этом величина набега фазы не зависит от длины нанотрубки и при больших значениях $n$ совпадает с адиабатическим инвариантом [20]

$$
I(\varepsilon)=\frac{1}{2 \pi} \oint p d x=\frac{\hbar \Delta \tilde{\chi}}{\pi}=\hbar(n+1 / 2),
$$

где интеграл по замкнутому контуру взят по полному периоду классического движения частицы. Условие (28) соответствует принципу квантования Бора-Зомерфельда старой квантовой теории. Уровни энергии в (27) обратно пропорциональны длине нанотрубки и совпадают со спектром одномерного осциллятора. Такая закономерность является естественной, если учесть, что в соответствии с результатами работы [18] движение носителей зарядов в нанотрубке сводится к уравнению Эрмита. В случае ограниченной области движения носителя заряда собственные функции выражаются через полиномы Эрмита, а собственные значения энергии образуют эквидистантный спектр. Следует отметить, что фаза в физических процессах является важной величиной. Например, взаимодействие двух солитонов характеризуется величиной сдвига фазы; сечение упругого и неупругого рассеяния нуклонов на ядре, фотонов на рассеивающих центрах также зависит от величины сдвига фазы; связь набега фазы с адиабатическим инвариантом (принцип квантования Бора-Зомерфельда). Если энергия электрона совпадает с уровнем энергии (27) стационарных состояний заряженных частиц в нанотрубке, то из (26) и (27) следует, что $\varphi_{0}=\pi / 2$. В этом случае коэффициент прохождения будет равен нулю. Действительно, из (26) следует $\sin ^{2}\left(k_{2} L_{1}-\varphi_{0}\right)=0$. С учетом равенства $\varphi_{0}=\arctan \left(\kappa / k_{2}\right)$ из (26) и условия $\varphi_{0}=\pi / 2$ получаем, что $k_{2} \rightarrow 0, \kappa \neq 0$. Из (21) следует, что при $k_{2} \rightarrow 0$ коэффициент прохождения стремится к нулю.

Так как выполняется условие $0<\varphi_{0}<\pi / 2$, наименьшее значение энергии $\varepsilon_{n}$, при которой выполняется $\sin ^{2}\left(k_{2} L_{1}-\varphi_{0}\right)=0$, достигается при выполнении равенства

$$
k_{2} L_{1}=\varphi_{0} \Leftrightarrow \kappa L_{1}=k_{2} L_{1} \tan \left(k_{2} L_{1}\right),
$$

где $\varphi_{0}=\arctan \left(\kappa / k_{2}\right)$. Из (23) с учетом (29) получаем выражение для максимального значения величины коэффициента прохождения

$$
\tau=\frac{4 \kappa k_{2}^{2}}{\left(\kappa^{2}+k_{2}^{2}\right) k_{1}} \exp \left[-\frac{4 \sqrt{2 m|e| W}}{3 \hbar}\left(L_{2}-L_{1}\right)^{3 / 2}\right] .
$$

При $k_{2}=k_{1}$ выражение (30) переходит в (25).

При $L_{2} \gg L_{1}$ в $(30)$ под экспонентой с учетом приближенного равенства $|e| W\left(L_{2}-L_{1}\right) \approx E$ сле- дует зависимость, аналогичная зависимости ФаулераНордгейма [22]

$$
\tau \sim D=\exp \left[-\frac{4 \sqrt{2 m|E|^{3}}}{3 \hbar} \frac{1}{|e| W}\right],
$$

где $D-$ коэффициент прозрачности.

\section{4. Влияние длины нанотрубки при полевой эмиссии на функцию Нордгейма}

При полевой эмиссии с поверхности металла (нанотрубки отсутствуют) коэффициент прозрачности $D$ в отличие от (31) содержит функцию Нордгейма $\theta(y)$ [10]

$$
D=\exp \left[-\frac{4 \sqrt{2 m|E|^{3}}}{3 \hbar} \frac{1}{|e| W} \theta(y)\right]
$$

в которой аргументом $y$ является относительное снижение работы выхода $\Delta(|e| \varphi)=\sqrt{|e|^{3} W}$ (в системе СГС), где работа выхода равна, внешним электрическим полем напряженностью в соответствии с эффектом Шотки ([10] (см. также [23])

$$
y=\frac{\Delta(|e| \varphi)}{|e| \varphi}=\frac{\sqrt{|e| W}}{\varphi} \approx \frac{|e| W L_{1}}{|e| W L_{2}}=\frac{L_{1}}{L_{2}} .
$$

В соответствии с (23) при наличии нанотрубки коэффициент прозрачности также определяется равенством (32) (см. также (30)), только вместо функции Нордгейма $\theta(y)$ нужно использовать функцию $\Theta(y)$

$$
\begin{gathered}
D=\exp \left[-\frac{4 \sqrt{2 m|E|^{3}}}{3 \hbar} \frac{1}{|e| W} \Theta(y)\right], \\
\Theta(y)=(1-y)^{3 / 2}, \quad y=\frac{L_{1}}{L_{2}} .
\end{gathered}
$$

В таблице приведена зависимость функции Нордгейма $\theta(y)$ и функции $\Theta(y)$, где функция $\Theta(y)$ и величина $y$ вычислялись в соответствии с формулой (34).

Зависимость функций $\theta(y)$ [23] и $\Theta(y)$ от величины $y$

\begin{tabular}{c|l|l|l|l|l|l|l|l|l|l|l}
\hline$y$ & 0 & 0.1 & 0.2 & 0.3 & 0.4 & 0.5 & 0.6 & 0.7 & 0.8 & 0.9 & 1 \\
\hline$\theta(y)$ & 1 & 0.98 & 0.94 & 0.87 & 0.79 & 0.68 & 0.58 & 0.45 & 0.31 & 0.16 & 0 \\
\hline$\Theta(y)$ & 1 & 0.85 & 0.72 & 0.59 & 0.46 & 0.35 & 0.25 & 0.16 & 0.09 & 0.03 & 0
\end{tabular}

С учетом (34), коэффициент прохождения из (23) можно записать в виде

$$
\begin{gathered}
\tau=\frac{2 \kappa k_{2}}{\kappa^{2}+} k_{2}^{2} \frac{2 k_{1} k_{2}}{k_{1}^{2}+\left(2 m / \hbar^{2}\right)\left(U_{1}-U_{2 a}\right) \sin ^{2}\left(k_{2} L_{1}-\varphi_{0}\right)} \\
\times \exp \left[-\frac{4 \sqrt{2 m|E|^{3}}}{3 \hbar} \frac{1}{|e| W} \Theta(y)\right],
\end{gathered}
$$


где величины $k_{1}$ и $k_{2}$ определены после формулы (14), а величина $\kappa$ определена в формуле (21)

$$
\kappa=\sqrt{2 m|e| W\left(L_{2}-L_{1}\right)} / \hbar=\sqrt{2 m|e| W L_{2}}[\Theta(y)]^{1 / 3} / \hbar .
$$

В (35) длина нанотрубки $L_{1}$ является постоянной величиной, а длина $L_{2}=-E /(|e| W)$ вычисляется в соответствии с формулой (10) (имеет место $L_{2}>L_{1}$ ).

В настоящей работе проведены расчеты по определению функции прохождения для различных нанотрубок нанометровой длины. Расчеты проводились в соответствии с формулой (23) (формула (23) аналогична формуле (35)). Чтобы понять различие в форме графиков на рис. 3, отдельно приведены результаты расчетов на рис. 4 и 5, где получены предэкспоненциальная функция прохождения и экспоненциальная часть (прозрачность $D(E))$.

На рис. 3 приведена зависимость коэффициента прохождения от энергии электронов для зигзаг-нанотрубок с металлическим типом проводимости, где кривым 1-4 соответствуют нанотрубки длиной $L_{1}=3.5,3.0,2.5$, $2.0 \mathrm{~nm}$. Величина напряженности поля $W=10^{9} \mathrm{~V} / \mathrm{m}$. В расчетах полагалось, что $U_{1}=-4 \mathrm{eV}, U_{2 a}=-7.6 \mathrm{eV}$. Остальные параметры приведены на рис. 3.

Различие в форме графиков можно объяснить с помощью рис. 4 и 5, на которых приведена зависимость предэкспоненциальной функции $\alpha(E)$ от энергии $E$. На врезке изображена зависимость функции прозрачности $D(E)$ от энергии в интервале энергий $-U_{1} \leq E<-|e| W L_{1}$. Из рисунков видно, что функция $\alpha$ равняется нулю в точках $E=-U_{1}$ и $E=-|e| W L_{1}$ (то же самое относится к функции прохождения $\tau$ из рис. 3). Поскольку при $E=-U_{1}$ и $E=-|e| W L_{1}$ величины $k_{1}$ и $\kappa$ равны нулю, в соответствии с (23) функции $\alpha$ и $\tau$ при таких значениях энергии равняются нулю $\tau=0$. Из рис. 3 следует, что при $L_{1}=3.5$ и $3.0 \mathrm{~nm}$ функция $\alpha(E)$ имеет один мак-

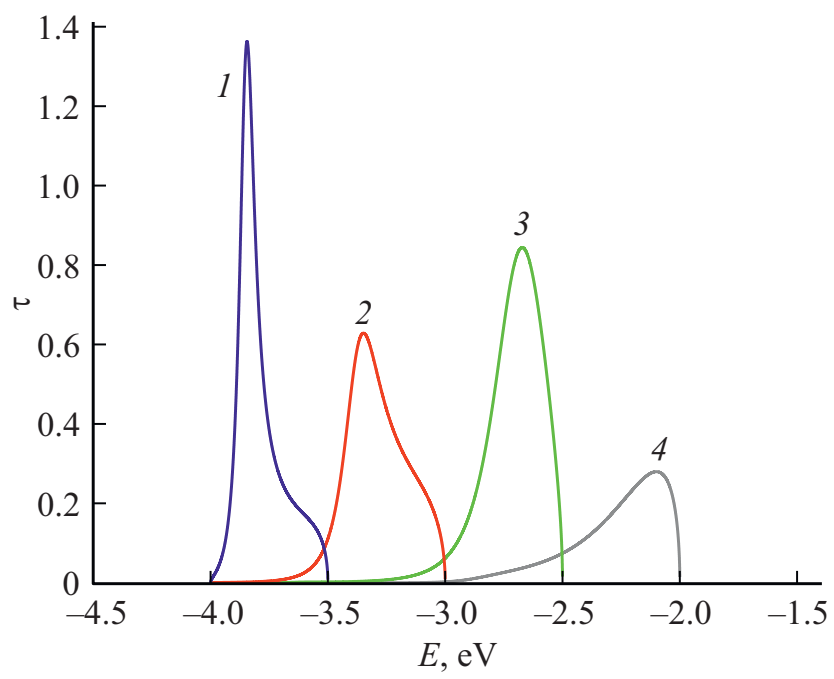

Рис. 3. Зависимость коэффициента прохождения от энергии электронов для нанотрубок при $-U_{1} \leq E L-|e| W L_{1}$. Кривые 1-4 соответствуют длинам нанотрубок.

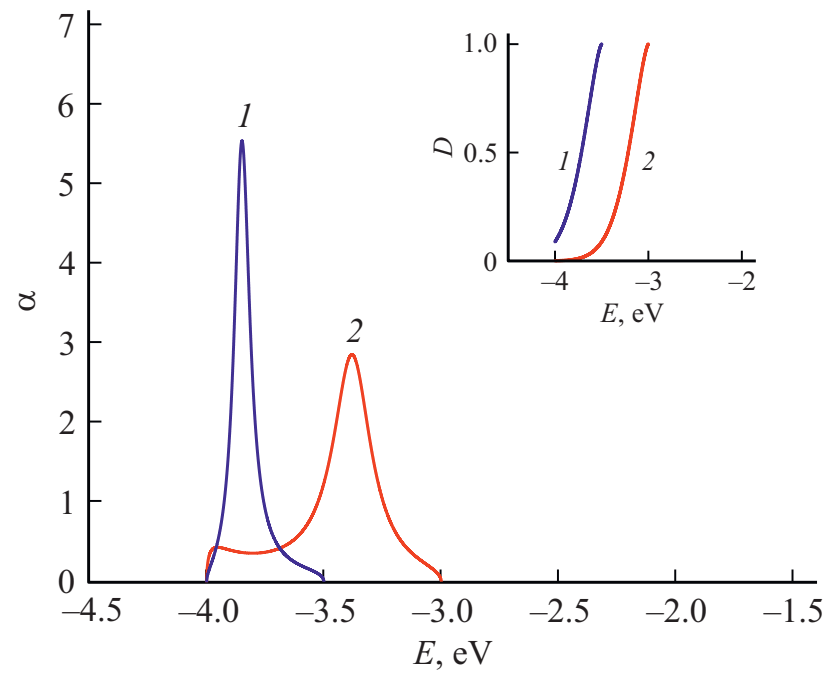

Рис. 4. Зависимость функции $\alpha$ от энергии электрона $E$. Кривая 1 соответствут случаю $L_{1}=3.5 \mathrm{~nm}$, кривая 2 соответствут случаю $L_{1}=3 \mathrm{~nm}$. На врезке изображена зависимость функции прозрачности $D$ от энергии. Кривые 1 и 2 также соответствуют случаю $L_{1}=3.5$ и $3 \mathrm{~nm}$.

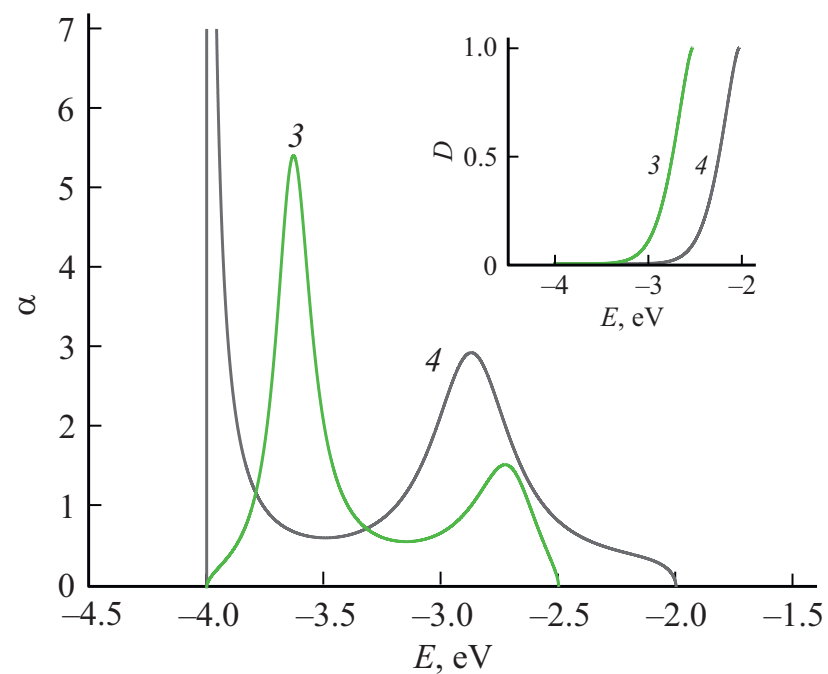

Рис. 5. Зависимость функции $\alpha$ от энергии $E$ электрона. Кривая 3 соответствут случаю $L_{1}=2.5 \mathrm{~nm}$, кривая 4 соответствут случаю $L_{1}=2 \mathrm{~nm}$. На врезке изображена зависимость функции прозрачности $D$ от энергии $E$. Кривые 3 и 4 также соответствуют случаю $L_{1}=2.5$ и $2 \mathrm{~nm}$.

симум, поэтому функция прохождения $\tau$ имеет также один максимум, причем максимумы функций $\alpha$ и $\tau$ при одинаковых длинах нанотрубок практически совпадают. Из рис. 5 следует, что при $L_{1}=2.5 \mathrm{~nm}$ предэкспоненциальная функция $\alpha$ имеет два максимума в точках $E_{1} \approx-3.4 \mathrm{eV}$ и $E_{2} \approx-2.7 \mathrm{eV}$, причем $\alpha\left(E_{2}\right)<\alpha\left(E_{1}\right)$. Поскольку значение экспоненциальной части функции прохождения в точке $E_{2}$ значительно больше, чем в точке $E_{1}$, то значение функции прохождения также в точке $E_{2}$ должно быть значительно больше, чем в 
точке $E_{1}$, что и подтверждается зависимостью функции $\tau$ на рис. 3 для кривой 3 . В случае $L_{1}=2.0 \mathrm{~nm}$ на рис. 5 существует один максимум, так же как и для функции прохождения на рис. 3 (кривая 4), но при этом максимумы на этих кривых не совпадают. Это объясняется тем, что „расстояние“ от правой точки области определения энергии $E=-|e| W L_{1}$ до единственного максимума очень большое. Поэтому функция прохождения смещена к правой границе кривой 4 на рис. 5.

\section{5. Обсуждение результатов}

Из рис. 3 следует, что на левой $E=U_{1}$ и правой $E=|e| W L_{1}$ границах области определения энергии $U_{1} \leq E<-|e| W L_{1}$ коэффициент прохождения равняется нулю. Это объясняется тем, что при выполнении условий $E=U_{1}$ и $E=-|e| W L_{1}$ в соответствии с (14) и (21) выполняются равенства $k_{12}=0$ и $\kappa=0$. Равенство нулю величины $k_{1}$ объясняется недостатком рассматриваемой модели. В частности, предполагается, что в области металла закон дисперсии определяется одномерной геометрией, в то время как в металле, например, в случае простой кубической решетки для координат атома нужно использовать трехмерный закон дисперсии [24]

$$
E=-\alpha=2 \gamma\left(\cos k_{x} a+\cos k_{y} a+\cos k_{z} a\right) .
$$

Поскольку наличие на электроде электрического потенциала приводит к изменению химического потенциала на электроде (см. (2)), на катоде величина $k_{1}$ будет отлична от нуля. Поскольку закон дисперсии (37) обобщает закон дисперсии одномерных [3] и двумерных наноструктур [25] в окрестности уровня Ферми, можно также использовать другую модель, в которой движение электронов на аноде можно аппроксимировать полииновой цепочкой или нанотрубками. Равенство нулю величины $\kappa=0$ на правой границе области определения энергии объясняется тем, что решение функции $\chi(z)$ из (12) является асимптотикой функции Эйри, и в окрестности точки $z=L_{2}$ это решение неверно. Тем фактом, что решение функции $\chi(z)$ из (12) является асимптотикой функции Эйри, можно также объяснить то, что коэффициент прохождения для случая $L_{1}=3.5 \mathrm{~nm}$ (кривая 1) больше единицы. Действительно, асимптотика функции Эйри (12) означает, что должно выполняться условие $|\xi| \gg 1$. Из рис. 3 следует, что для кривой 1 точка максимума равна $E_{\max }=-3.8 \mathrm{eV}$. Из (10) следует $L_{2}=\left|E_{\max }\right| /(|e| W)=3.8 \mathrm{~nm}$, $\xi=\left(2 m|e| W / \hbar^{2} \mid\right)^{1 / 3}\left(L_{2}-L_{1}\right) \approx 0.9 \quad$ (условие $\quad|\xi| \gg 1$ не выполняется), т.е. в окрестности точки $z=L_{2}$ решение (12) для функции Эйри неверно, значит полученное решение для функции прохождения (на рис. 3, кривая 1) будет нефизично. Для более точного решения в этом случае нужно использовать точное решение для функции Эйри $[15,16]$.
Следует также отметить, что в области $0 \leq z \leq L_{1}$ следует использовать собственные функции и собственные значения энергии в удлиненных нанотрубках $[17,18]$ или нанолентах [19], полученные из модели двухточечной и четырехточечной элементарных ячеек.

\section{Заключение}

Функция $\Theta(y)$, которая входит в выражение (34) для функции прозрачности $D$, выполняет роль, аналогичную функции Нордгейма, причем аргумент функции $y=L_{1} / L_{2}=|e| W L_{1} /|E|$ зависит как от энергии заряженной частицы, так и от длины нанотрубки и напряженности поля. Наличие функции $\Theta(y)$ позволяет оценить зависимость величины коэффициента прозрачности от длины нанотрубки. При $W=10^{9} \mathrm{~V} / \mathrm{m}$, $E_{2}=-|e| W L_{2}=-3.85 \mathrm{eV},|e| W L_{1}=-3.5 \mathrm{eV}$ (рис. 3,4 ) следует $L_{1} / L_{2}=1 / 1.1 \approx 0.9$. Из $(33)$ следует $y=\Delta(|e| \varphi)=\sqrt{|e| W} / \varphi \approx L_{1} / L_{2}$. Из таблицы при $y=0.9$ получаем $\Theta \approx 0.03, \theta \approx 0.16$. Из равенства $(32)$ получаем, что отношение прозрачностей $D_{\Theta} / D_{\theta} \approx 806$, где прозрачности $D_{\theta}$ и $D_{\Theta}$ определяются функцией Норгейма $\theta(y)$ и функцией $\Theta(y)$ в формуле (34). Поскольку подынтегральное выражение для тока $I$ в формуле Ландауэра (2) линейно зависит от коэффициента прозрачности (от коэффициента прохождения), условие $D_{\Theta} \gg D_{\theta}$ должно привести к значительному увеличению тока при полевой эмиссии по сравнению полевой эмиссии с поверхности металла при прочих одинаковых условиях, либо можно использовать меньшее значение электрического поля. Величина коэффициента прозрачности увеличивается при $y=L_{1} / L_{2} \rightarrow 1$, где $y<1$.

Коэффициент прохождения за счет экспоненциального убывания от энергии коэффициента прозрачности (рис. 4,5) имеет точку максимума в окрестности $-0.5 \mathrm{eV}-|e| W L_{2} \leq E \leq-|e| W L_{2}$ энергии электрона. Возможно, такая закономерность сохранится при увеличении длины нанотрубки до микрометровой длины $L_{12} \leq 1 \mu \mathrm{m}$ (это условие существования баллистического транспорта, т.е. применимости формулы Ландауэpa (2)).

Как величина набега фазы $\Delta \varphi$, которая была получены исходя из закона дисперсии для свободных электронов, так и величина набега фазы $\Delta \tilde{\chi}$ в зигзаг-нанотрубке и крессельных нанолентах при наличии продольного постоянного электрического поля, которая была получена на основе модели двухточечной и четырехточечной элементарных ячеек [17-19], приведут к периодической зависимости функции прохождения электронов от энергии, что характерно для периодических структур [26]. Это означает, что полученные в работе закономерности будут иметь место, если использовать модель двухточечной и четырехточечной элементарных ячеек.

Следует подчеркнуть, что использование модели двухточечной и четырехточечной элементарных ячеек приводит к тому, что волновые функции носителей за- 
ряда в нанотрубках и узких нанолентах аналогичны спиновым функциям майорановских фермионов [1719,27], в то время как электроны в вакууме являются фермионами Дирака. Это означает, что на границе раздела нанотрубка-вакуум коэффициент прохождения будет зависеть также от спиновых функций фермионов Майорана и Дирака.

Способность эмиттера к усилению электрического поля характеризуется коэффициентом полевого усиления $\beta$, определяемым как отношение локального значения напряженности электрического поля на наконечнике к среднему значению поля между электродами [28]. Основным геометрическим параметром, определяющим величину коэффициента усиления индивидуальной нанотрубки, является ее аспектное отношение $M=L_{1} / d$, где $d$ - диаметр нанотрубки. Рассмотрение, основанное на качественном анализе электростатической задачи, приводит к линейной пропорциональности между коэффициентом усиления и аспектным отношением нанотрубки при большом размере межэлектродного промежутка $H \gg L_{1}$. Поэтому в случае полевой эмиссии с поверхности металла аспектное отношение стараются увеличить. Для микрометровых нанотрубок аспектное отношение порядка 1000. В настоящей работе рассмотрены наночастицы в несколько нанометров, у которых величина аспектного отношения порядка десяти, поэтому, возможно, качественной аналогии с эффектом локального усиления электрического поля на эмиссионном центре по отношении к геометрическому значению поля не будет.

\section{Финансирование работы}

Н.Р. Садыков благодарит Национальный исследовательский ядерный университет МИФИ за поддержку в рамках проекта „Российская академическая подготовка“ (контракт № 02.a03.21.0005.27.08.2).

\section{Конфликт интересов}

Авторы заявляют, что у них нет конфликта интересов.

\section{Список литературы}

[1] A.Y. Kitaev. Phys. Usp., 44, 131 (2001).

[2] S. Hino, Y. Okada, K. Iwasaki, M. Kijima, H. Shirakawa. Chem. Phys. Lett., 372, 59 (2003).

[3] P.N. D'yachkov, V.A. Zaluev, E.Yu. Kocherga, N.R. Sadykov. J. Phys. Chem. C, 117, 16306 (2013).

[4] S. Eisler, A.D. Slepkov, E. Elliott, T. Luu, R. McDonald, F. Hegmann, R. Tykwinski. J. Am. Chem. Soc., 127, 2666 (2005).

[5] R. Saito, G. Dresselhaus, M.S. Dresselhaus. Physical Properties of Carbon Nan-otubes (Imperial College Press, London, 1998)

[6] C.T. White, J. Li, D. Gunlycke, J.W. Mintmire. Nano Lett., 7, 825 (2007). https://doi.org/10.1021/n10627745
[7] K. Wakabayashi, K.-I. Sasaki, T. Nakanishi, T. Enoki. Sci. Technol. Adv. Mater., 11, 054504 (2010). https://doi.org/10.1088/1468-6996/11/5/054504.

[8] П.Н. Дьячков. Электронные свойства и применение нанотрубок (БИНОМ, Лаборатория знаний, М., 2011)

[9] C. Rutherglen, P. Burke. Small., 5 (8), 884 (2009).

[10] А.В. Елецкий. УФН, 18 (9), 897 (2010).

[11] K.B.K. Teo, E. Minoux, L. Hudanski, F. Peauger, J.P. Schnell, L. Gangloff, P. Legagneux, D. Dieumegard, G.A.J. Amaratunga, W.I. Milne. Nature, 437, 968 (2005).

[12] A. Vul, K. Reich, E. Eidelman, M.L. Terranova, A. Ciorba, S. Orlanducci, V. Sessa, M.A. Rossi. Adv. Sci. Lett., 3, 110 (2010). https://doi.org/10.1166/ as1.2010.1104

[13] R. Landauer. Phil. Mag., 21, 863 (1972).

[14] M. Buttiker. Phys. Rev. B, 46, 12485 (1992).

[15] А.Ф. Никифоров, В.Б. Уваров. Специальные функции математической физики (Физматлит, М., 1978). [A.F. Nikiforov, V.B. Uvarov. Special Functions of Mathematical Physics [in Russian] (Birkhauser, Basel, 1988)]

[16] Г.Ф. Друкарев. Квантовая механика: учебное пособие (Изд-во ЛГУ, Л., 1988), ISBN 5-283-00292

[17] Н.Р. Садыков, Н.А. Скоркин. ЖТФ, 83 (5), 1 (2013). DOI: $10.1134 / \mathrm{S} 1063784213050186$

[18] Н.Р. Садыков. Теоретическая и математическая физика, 180 (3), 368 (2014).

[19] N.R. Sadykov, E.T. Muratov, I.A. Pilipenko, A.V. Aporoski. Physica E: Low-dimensional Systems and Nanostructures, 120, 114071 (2020). DOI: 10.1016/j.physe.2020.114071

[20] Л.Д. Ландау, Е.М. Лифшиц. Теоретическая фбизика, Квантовая механика. Нерелятивистская теория (Наука, М., 2002), т. 3. [L.D. Landau, E.M. Lifshitz. Course of Theoretical Physics [in Russian], v. 3, Quantum Mechanics: Non-Relativistic Theory (Nauka, Moscow, 2002); English transl. prev. ed., Pergamon, London (1958)]

[21] В.М. Галицкий, Б.М. Карнаков, В.И. Коган. Задачи по квантовой механике: учебное пособие (Наука, М., 1981)

[22] R.H. Fowler, L. Nordheim. Proc. Roy. Soc., 109 (A781), 173 (1928).

[23] А.П. Бабичев, Н.А. Бабушкина, А.М. Братковский, М.Е. Бродов, М.В. Быстров, Б.В. Виноградов, Л.И. Винокурова, Э.П. Гельман, А.П. Геппе, И.С. Григорьев, К.Г. Гуртовой, В.С. Егоров, А.В. Елецкий, Л.К. Зарембо, В.Ю. Иванов, В.Л. Ивашинцева, В.В. Игнатьев, Р.М. Имамов, А.В. Инюшкин, Н.В. Кадобнова, И.И. Карасик, К.А. Кикоин, В.А. Криворучко, В.М. Кулаков, С.Д. Лазарев, Т.М. Лифшиц, Ю.Э. Любарский, С.В. Марин, И.А. Маслов, Е.3. Мейлихов, А.И. Мигачев, С.А. Миронов, А.Л. Мусатов, Ю.П. Никитин, Л.А. Новицкий, А.И. Обухов, В.И. Ожогин, Р.В. Писарев, Ю.В. Писаревский, В.С. Птускин, А.А. Радциг, В.П. Рудаков, Б.Д. Сумм, Р.А. Сюняев, М.Н. Хлопкин, И.Н. Хлюстиков, В.М. Черепанов, А.Г. Чертов, В.Г. Шапиро, В.М. Шустряков, С.С. Якимов, В.П. Яновский. Физические величины: справочник, под. ред. И.С. Григорьева, Е.3. Мейлихова. (Энергоиздат, М., 1991), ISBN 5-283-04013-5

[24] Ч. Киттель. Введение в физику твердого тела (Наука, M., 1978) [C. Kittel. Introduction to Solid State Physics, 1953 (1st ed.), 2005 (8th ed.), ISBN 0-471-41526-X] 
[25] R. Saito, M. Fujita, G. Dresselhaus, M.S. Dresselhaus. Phys. Rev. B, 46, 1804 (1992).

[26] P.N. D’yachkov, V.A. Zaluev, E.Yu. Kocherga, N.R. Sadykov. J. Phys. Chem. C, 117, 16306 (2013).

[27] N.R. Sadykov, D.A. Peshkov, A.V. Aporoski, M.B. Belonenko. Int. J. Mod. Phys. B, 34 (21), 2050202 (2020).

DOI: $10.1142 / \mathrm{S} 0217979220502021-$

[28] М.Д. Бельский, Г.С. Бочаров, А.В. Елецкий, Т.J. Sommerer. ЖТФ, 80 (2), 130 (2010). 